Comment. Math. Helv. 76 (2001) 161-182

$0010-2571 / 01 / 010161-22 \$ 1.50+0.20 / 0$

(C) 2001 Birkhäuser Verlag, Basel

Commentarii Mathematici Helvetici

\title{
The group of self-distributivity is bi-orderable
}

Patrick Dehornoy

\begin{abstract}
We prove that the group of left self-distributivity, a cousin of Thompson's group $F$ and of Artin's braid group $B_{\infty}$ that describes the geometry of the identity $x(y z)=(x y)(x z)$, admits a bi-invariant linear ordering. To this end, we define a partial action of this group on finite binary trees that preserves a convenient linear ordering.
\end{abstract}

Keywords. Ordered groups, groups acting on trees, self-distributivity, Thompson's groups.

Mathematics Subject Classification (2000). 20F60, 20E08, 20 N02.

There exists a close connection between Thompson's group $F$ of [22], [17] and [1], and the associativity identity. Indeed, $F$ acts on bracketed expressions by moving the brackets, i.e., by applying associativity, and, conversely, every application of associativity comes from the action of an element of $F$. Thus, $F$ can be called the geometry group of associativity, as it captures a number of specific geometrical properties of that identity, in particular those expressed in the well-known MacLane-Stasheff pentagon relation [6] [16].

When we replace the associativity identity $x(y z)=(x y) z$ with the left selfdistributivity identity $x(y z)=(x y)(x z)$, Thompson's group $F$ is no longer relevant, but there exists another group $G_{L D}$ that similarly captures the geometrical aspects of the identity. The group $G_{L D}$ happens to be an extension of Artin's braid group $B_{\infty}$, of which it can be seen as a sort of tree version, a relation that explains the deep connection between braids and the self-distributive law. In the recent years, several new results about braids, in particular the existence of a linear ordering compatible with the product, have been discovered by projecting results initially established in $G_{L D}$ [4], leading in turn to a number of further developments [7], [15], [10], [21] — see [13]. Thus the group $G_{L D}$ (which will be defined by an explicit presentation below) may appear as an interesting object of study.

Order properties have been recently established for various groups connected with topology: besides the orderability of braid groups alluded to above, the orderability of the mapping class groups of surfaces with a nonempty boundary [20], the bi-orderability of the pure braid groups [14], the fact that Artin's braid groups are not bi-orderable in a strong sense [19]. Let us also mention work in progress by D. Rolfsen and B. Wiest about the orderability of knot groups. As for Thompson's 
group $F$, it can be realized as a group of diffeomorphisms of a real interval [12], and, as such, it acts on the reals, which easily implies that it is orderable, and even bi-orderable as shows the explicit form of the action [1].

As the group $G_{L D}$ is closely connected both with Thompson's group $F$ and with Artin's braid group $B_{\infty}$, the question of whether it is bi-orderable, like $F$, or not bi-orderable, like $B_{\infty}$, appears natural. It had been shown in [4] that $G_{L D}$ is equipped with a linear left-invariant preordering (which projects on the canonical left-invariant linear ordering of the braids). However, this preordering is not an ordering, and it is not right invariant, so it does not answer the above question. In this paper, we shall prove that, as for orderability, $G_{L D}$ is similar to $F$, and not to $B_{\infty}$ :

Proposition. The group $G_{L D}$ is bi-orderable, i.e., there exists a linear ordering on $G_{L D}$ that is compatible with product on both sides.

Our proof consists in defining an action of $G_{L D}$ that is reminiscent of the action of $F$ on the reals. However, due to an essential technical difference between associativity and self-distributivity, namely the fact that the variable $x$ is repeated twice in the right-hand term of the identity $x(y z)=(x y)(x z)$, there is no natural way to let $G_{L D}$ act on the reals via diffeomorphisms. Instead we shall let $G_{L D}$ act on finite binary rooted trees and observe that this action preserves some linear ordering of such trees. A similar approach is also possible in the case of Thompson's group $F$, in which case one essentially re-obtains the action of $F$ on $\mathbf{R}$, and, more generally, in the case of analog groups that can be associated with algebraic identities preserving the order of the variables [9].

The organization of the paper is as follows. In Section 1, we recall the definition of the group $G_{L D}$ and introduce its partial action on finite binary trees and, more generally, on terms, which are finite binary trees with labeled leaves. In Section 2, we construct a linear ordering of terms connected with their coding by words using the left Polish form. In Section 3, we show that the action of $G_{L D}$ on terms preserves the previous ordering, and we deduce a bi-invariant ordering on $G_{L D}$. Finally, in Section 4, we deduce from the action of $G_{L D}$ on finite trees an action of the positive part of $G_{L D}$-a certain submonoid of $G_{L D}$ of which $G_{L D}$ is the groupe of fractions - on the Cantor line and on the reals.

\section{The action of $G_{L D}$ on terms}

In this preliminary section, we recall the definition of the group $G_{L D}$, and its connections with the left self-distributivity identity, with Thompson's group $F$, and with Artin's braid group $B_{\infty}$. We also define a partial action of $G_{L D}$ on terms connected with the left self-distributivity identity. 


\section{The group $G_{L D}$}

The group $G_{L D}$ is a countable group that describes, in some sense explained below, the geometry of the left self-distributivity identity

$$
x(y z)=(x y)(x z)
$$

We shall define $G_{L D}$ using an explicit presentation. The generators are in oneto-one correspondence with the vertices in a complete binary rooted tree: so we can specify a generator by using a finite sequence of 0 's and 1's describing the path from the root to the considered vertex. Such finite sequences will be called addresses; we use $A$ for the set of all addresses, and $\phi$ for the empty address, i.e., the address of the root (Figure 1.1). For $\alpha, \beta \in A, \alpha \beta$ denotes the concatenation of $\alpha$ and $\beta$. We say that two addresses $\alpha, \beta$ are orthogonal, written $\alpha \perp \beta$, if there exists an adress $\gamma$ such that $\gamma 0$ is a prefix of $\alpha$ and $\gamma 1$ is a prefix of $\beta$, or vice versa.

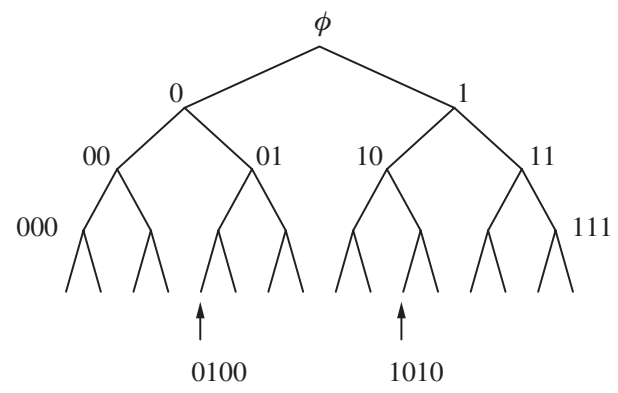

Figure 1.1. Binary addresses.

Definition. We denote by $G_{L D}$ the group $\left\langle\left\{g_{\alpha} ; \alpha \in A\right\} ; R_{L D}\right\rangle$, where $R_{L D}$ consists of the following five families of relations:

$$
\begin{array}{rlrl}
g_{\alpha} \cdot g_{\beta} & =g_{\beta} \cdot g_{\alpha} \quad \text { for } \alpha \perp \beta, & (\text { type } \perp) \\
g_{\alpha 0 \beta} \cdot g_{\alpha} & =g_{\alpha} \cdot g_{\alpha 10 \beta} \cdot g_{\alpha 00 \beta}, & (\text { type } 0) \\
g_{\alpha 10 \beta} \cdot g_{\alpha} & =g_{\alpha} \cdot g_{\alpha 01 \beta}, & & (\text { type 10) } \\
g_{\alpha 11 \beta} \cdot g_{\alpha} & =g_{\alpha} \cdot g_{\alpha 11 \beta}, & (\text { type 11) } \\
g_{\alpha 1} \cdot g_{\alpha} \cdot g_{\alpha 1} \cdot g_{\alpha 0} & =g_{\alpha} \cdot g_{\alpha 1} \cdot g_{\alpha} . & & (\text { type 1) }
\end{array}
$$

Let us recall that Artin's braid group $B_{\infty}$ can be defined as the group generated by an infinite sequence $\sigma_{1}, \sigma_{2}, \ldots$ subject to the relations

$$
\begin{aligned}
\sigma_{i} \cdot \sigma_{j} & =\sigma_{j} \cdot \sigma_{i} \quad \text { for }|i-j| \geq 2, & & \text { type (i) } \\
\sigma_{i+1} \cdot \sigma_{i} \cdot \sigma_{i+1} & =\sigma_{i} \cdot \sigma_{i+1} \cdot \sigma_{i} . & & \text { type (ii) }
\end{aligned}
$$


Then, from the presentation, it is obvious that the mapping

$$
\operatorname{pr}: g_{\alpha} \mapsto \begin{cases}\sigma_{i} & \text { for } \alpha=1^{i-1} \text { (i.e., } 1 \text { repeated } i-1 \text { times), } \\ 1 & \text { if } \alpha \text { contains at least one } 0\end{cases}
$$

defines a surjective homomorphism of $G_{L D}$ onto $B_{\infty}: B_{\infty}$ is what remains from $G_{L D}$ when we collapse every generator associated with a vertex of the complete binary tree not lying on the right branch. As $B_{\infty}$ is not finitely generated, $G_{L D}$ is not either finitely generated. The kernel of the projection of $G_{L D}$ onto $B_{\infty}$ is large (and complicated): if $H_{i}$ denotes the parabolic subgroup of $G_{L D}$ generated by all generators $g_{\alpha}$ with $\alpha$ beginning with $1^{i} 0$, then, by type $\perp$ relations, the elements of $H_{i}$ and $H_{j}$ commute for $i \neq j$, and $\operatorname{Ker}(\mathrm{pr})$ includes the direct product $H_{0} \times$ $H_{1} \times \cdots$. It can then be shown that, for every $i$, the mapping $g_{\alpha} \mapsto g_{1^{i} 0 \alpha}$ induces an isomorphism of $G_{L D}$ onto $H_{i}$. More generally, a parabolicity theorem asserts that, for every address $\gamma$, mapping $g_{\alpha}$ to $g_{\gamma \alpha}$ defines an isomorphism of $G_{L D}$ onto the subgroup of $G_{L D}$ generated by those generators $g_{\beta}$ such that $\beta$ begins with $\gamma$.

The syntactic form of the relations $R_{L D}$ defining $G_{L D}$ is reminiscent of the Coxeter relations that define Artin groups, though they do not preserve the length and are not symmetric. It is proved in [4] and [5] that most of the tools developed by Garside in his study of braid groups [11] can be extended to groups defined by such generalized Coxeter relations. The specific case of $G_{L D}$ is made difficult by the fact that, in contradistinction to $B_{\infty}, G_{L D}$ is not the inductive limit of an increasing family of groups of finite type. However, by introducing local counterparts to Garside's fundamental braids $\Delta_{n}$, one can extend some of the results, and, in particular, prove that $G_{L D}$ is a group of fractions:

Proposition 1.1. [4], [8] Let $G_{L D}^{+}$be the submonoid of $G_{L D}$ generated by the elements $g_{\alpha}$ with $\alpha \in A$. Then every element of $G_{L D}$ can be written as $a b^{-1}$ with $a, b \in G_{L D}^{+}$.

We claim nothing about the presentation of the monoid $G_{L D}^{+}$: whether $G_{L D}^{+}$admits, as a monoid, the above presentation of $G_{L D}$ is currently unknown.

\section{Terms and trees}

Terms will play a central role in the sequel. Several equivalent definitions are possible. For our current purpose, it will be convenient to consider terms as finite trees.

Definition. Let $x_{1}, x_{2}, \ldots$ be a fixed sequence of variables (= letters); a term is defined to be a finite binary rooted tree whose leaves (i.e., vertices of degree 1) wear labels in $\left\{x_{1}, x_{2}, \ldots\right\}$. We write $T_{\infty}$ for the set of all terms, and $T_{1}$ for the subset of $T_{\infty}$ consisting of those terms where all leaves are labeled $x_{1}$. 
Thus,
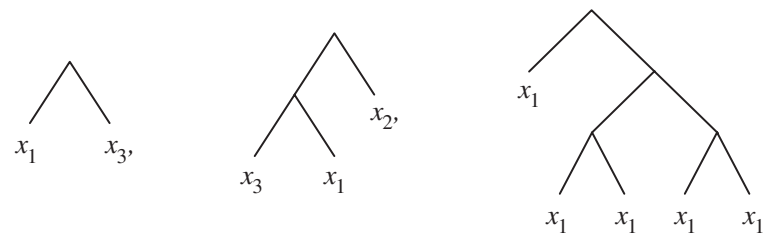

are typical terms in $T_{\infty}$. The latter belongs to $T_{1}$. In the case of $T_{1}$, we can of course forget about the labels, and identify a term with an unlabeled tree.

Terms are equipped with a natural product, namely the operation that associates with two terms $t_{0}, t_{1}$ the term, denoted $t_{0} \cdot t_{1}$, consisting of a root with two successors, a left one which is $t_{0}$, and a right one which is $t_{1}$ :

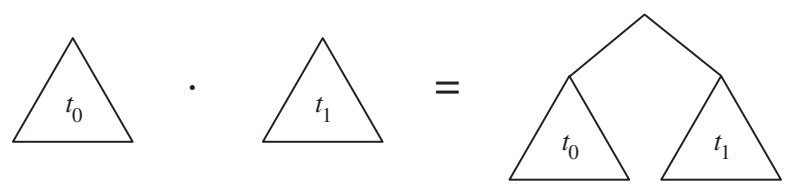

Then, provided we identify the variable $x_{i}$ with the tree consisting of a single vertex labeled $x_{i},\left(T_{\infty}, \cdot\right)$ is a free magma based on $\left\{x_{1}, x_{2}, \ldots\right\}$, and $\left(T_{1}, \cdot\right)$ is a free magma based on $\left\{x_{1}\right\}$.

Each vertex in a finite binary rooted tree can be specified by an address in $A$ describing the path from the root to that vertex. For $t$ a term, we define the outline of $t$ to be the collection of all addresses of leaves in (the tree associated with) $t$, and the skeleton of $t$ to be the collection of the addresses of vertices in $t$ : thus, for instance, the outline of the term $\left(x_{3} \cdot x_{1}\right) \cdot x_{2}$ is the set $\{00,01,1\}$, while its skeleton is $\{00,01,0,1, \phi\}$, as $t$ comprises three leaves and two inner vertices.

For $t$ a term, and $\alpha$ an address in the skeleton of $t$, we have the natural notion of the $\alpha$-subterm of $t$, denoted $\operatorname{sub}(t, \alpha)$ : this is the subtree of $t$ whose root lies at address $\alpha$. This amounts to defining inductively

$$
\operatorname{sub}(t, \alpha)= \begin{cases}t & \text { if } t \text { is a variable or } \alpha=\lambda \text { holds } \\ \operatorname{sub}\left(t_{0}, \beta\right) & \text { for } t=t_{0} \cdot t_{1} \text { and } \alpha=0 \beta \\ \operatorname{sub}\left(t_{1}, \beta\right) & \text { for } t=t_{0} \cdot t_{1} \text { and } \alpha=1 \beta\end{cases}
$$

For instance, the 0 -subterm of the term $\left(x_{3} \cdot x_{1}\right) \cdot x_{2}$ is the term $x_{3} \cdot x_{1}$, its 01-subterm is the term $x_{1}$, while its 010 -subterm is not defined. Observe that the outline of a term $t$ is the set of those addresses $\alpha$ such that $\operatorname{sub}(t, \alpha)$ is a variable. 


\section{The action of $G_{L D}$ on terms}

We shall now describe the connection between the group $G_{L D}$ and the left selfdistributivity identity by means of a partial actions of $G_{L D}$ on terms.

In the sequel, a set equipped with a left self-distributive operation will be called an LD-system (the names LD-magma and LD-groupoid have also been used occasionally). Let us say that two terms $t, t^{\prime}$ in $T_{\infty}$ are $L D$-equivalent, denoted $t={ }_{L D} t^{\prime}$, if we can transform $t$ to $t^{\prime}$ by repeatedly applying Identity $(L D)$. By standard arguments, the quotient structure $T_{\infty} /=_{L D}$ is a free LD-system based on $\left\{x_{1}, x_{2}, \ldots\right\}$, and studying free LD-systems amounts to studying LD-equivalence of terms.

Applying the left self-distributivity identity to a term $t$ consists in replacing some subterm of $t$ which has the form $t_{1} \cdot\left(t_{2} \cdot t_{3}\right)$ with the corresponding term $\left(t_{1} \cdot t_{2}\right) \cdot\left(t_{1} \cdot t_{3}\right)$, or vice versa. Having defined the $\alpha$-subterm of a term precisely, we can take into account the position, i.e., the address, of the subterm where the identity is applied. This leads to defining a partial action on $T_{\infty}$ of the free monoid $\left(A \cup A^{-1}\right)^{*}$ generated by $A$ and a disjoint copy $A^{-1}$ of $A$ comprising a formal inverse $\alpha^{-1}$ for each address $\alpha$.

Definition. (i) For $t$ a term, and $\alpha$ an address such that the $\alpha$-subterm of $t$ exists and can be written as $t_{1} \cdot\left(t_{2} \cdot t_{3}\right)$, we define $(t) \alpha$ to be the term obtained from $t$ by replacing the $\alpha$-subterm with the corresponding term $\left(t_{1} \cdot t_{2}\right) \cdot\left(t_{1} \cdot t_{3}\right)$.

(ii) For $t$ a term, and $\alpha$ an address, we define $(t) \alpha^{-1}$ to be the unique term $t^{\prime}$ verifying $t=\left(t^{\prime}\right) \alpha$, when it exists.

(iii) For $t$ a term, and $w$ a word on $A \cup A^{-1}$, say $w=\alpha_{1}^{e_{1}} \cdot \ldots \cdot \alpha_{p}^{e_{p}}$, with $\alpha_{i} \in A$ and $e_{i}= \pm 1$, we define $(t) w$ to be $\left(\ldots\left((t) \alpha_{1}^{e_{1}}\right) \alpha_{2}^{e_{2}} \ldots\right) \alpha_{p}^{e_{p}}$, when it exists.

Thus $(t) \alpha$ is the term obtained by expanding $t$ at $\alpha$ using left self-distributivity:
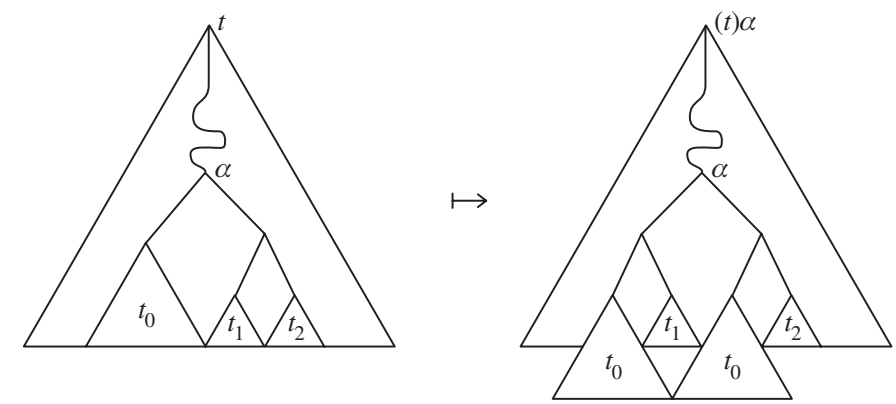

Example 1.2. Let $t=x_{1} \cdot x_{2} \cdot x_{3} \cdot x_{4}$-here, and everywhere in the sequel, we take the convention that missing brackets are to be added on the right, so, for instance, the previous expression stands for $x_{1} \cdot\left(x_{2} \cdot\left(x_{3} \cdot x_{4}\right)\right)$ - then the only addresses $\alpha$ for which $(t) \alpha$ exists are $\lambda$ and 1 , and we have $(t) \phi=\left(x_{1} \cdot x_{2}\right) \cdot\left(x_{1} \cdot x_{3} \cdot x_{4}\right)$, and $(t) 1=$ $x_{1} \cdot\left(x_{2} \cdot x_{3}\right) \cdot\left(x_{2} \cdot x_{4}\right)$. 
By construction, the term $(t) \alpha$ is defined if and only if the address $\alpha 10$ belongs to the skeleton of $t$, and that $(t) \alpha^{-1}$ exists if and only if the addresses $\alpha 00$ and $\alpha 10$ belong to the skeleton of $t$, and, in addition, $\operatorname{sub}(t, \alpha 10)=\operatorname{sub}(t, \alpha 00)$ holds.

We thus have obtained a partial right action of the free monoid $\left(A \cup A^{-1}\right)^{*}$ on the set $T_{\infty}$. By construction, we have:

Lemma 1.3. Two terms $t, t^{\prime}$ in $T_{\infty}$ are LD-equivalent if and only if $t^{\prime}=(t) w$ holds for some word $w$ in $\left(A \cup A^{-1}\right)^{*}$.

The previous action is partial, i.e., not everywhere defined, in essence. In particular, there exist words $w$ such that $(t) w$ is defined for no term $t$ : this happens for instance for $w=\phi \cdot 1 \cdot \phi^{-1}$, as, by construction, no term of the form $(t) \phi \cdot 1$ may have equal subterms at 00 and 10 , hence be eligible for the action of $\phi^{-1}$. This situation is unpleasant, but - in contradistinction to easier cases like the case of associativity - there exists no way of avoiding it by using a convenient quotient or subset, or by replacing groups by groupoids (small categories with inverse).

By definition, the group $G_{L D}$ is a quotient of the free group generated by the $g_{\alpha}$ 's, $\alpha \in A$, hence of the free monoid $\left(A \cup A^{-1}\right)^{*}$ : for $w$ a word on $A \cup A^{-1}$, we denote by $\bar{w}$ the image of $w$ in $G_{L D}$ under the homomorphism that maps $\alpha$ to $g_{\alpha}$ and $\alpha^{-1}$ to $g_{\alpha}^{-1}$.

The connection between $G_{L D}$ and left self-distributivity comes from the fact that the partial action of $\left(A \cup A^{-1}\right)^{*}$ on terms described above factors through $G_{L D}$ and the resulting action is faithful in the following sense:

Proposition 1.4. [4] Assume that $w, w^{\prime}$ are words on $A \cup A^{-1}$ and there exists at least one term $t$ such that both $(t) w$ and $(t) w^{\prime}$ are defined Then the following are equivalent:

(i) There exists at least one term $t$ satisfying $(t) w=(t) w^{\prime}$;

(ii) For every term $t$ such that $(t) w$ and $(t) w^{\prime}$ exist, we have $(t) w=(t) w^{\prime}$;

(iii) The words $w$ and $w^{\prime}$ represent the same element of $G_{L D}$.

In the particular case when $w$ and $w^{\prime}$ are words on $A$, the condition that there exists at least one term $t$ such that both $(t) w$ and $(t) w^{\prime}$ are defined is always satisfied.

The previous statements may appear convoluted, but, because there exist words $w$ such that $(t) w$ is defined for no $t$, there is no way to obtain a simpler statement: the action of $\alpha^{-1}$ is not an exact inverse of the action of $\alpha$, as $(t) \alpha \cdot \alpha^{-1}=t$ holds only if $(t) \alpha$ is defined. The proof of Proposition 1.4 is delicate: as one can expect, it is not very difficult to check that (iii) implies (ii), i.e., that the action factors through $G_{L D}$, but proving that (i) implies (iii), i.e., that the factorized action is faithful, requires a nontrivial argument.

Owing to the previous result, we obtain a well-defined partial action of $G_{L D}$ on $T_{\infty}$ : for $t$ a term, and $a$ in $G_{L D}$, we define $(t) a$ to be $(t) w$ where $w$ is any word on $A \cup A^{-1}$ that represents $a$ and is such that $(t) w$ exists, if such a word exists. 
The action is partial, as there exist some elements $a$ of $G_{L D}$, like $g_{\phi} g_{1} g_{\phi}^{-1}$, such that $(t) w$ exist for no expression $w$ of $a$, but it is well-defined in the sense that, if $w$ and $w^{\prime}$ are distinct expressions of some element $a$ such that both $(t) w$ and $(t) w^{\prime}$ exist, then the latter terms are equal.

Lemma 1.3 and Proposition 1.4 immediately yield:

Proposition 1.5. For every term $t$, the LD-equivalence class of $t$ is the orbit of $t$ under the (partial) action of $G_{L D}$, and this action is faithful: we have $t^{\prime}=_{L D} t$ if and only if $t^{\prime}=(t) a$ holds for some $a$ in $G_{L D}$, and, in this case, the involved element a is unique.

This statement should make it natural to call $G_{L D}$ the geometry group of Identity $(L D)$.

\section{The connection between $G_{L D}$ and Thompson's group $F$}

A similar approach can be developed when left self-distributivity is replaced with associativity. This amounts to considering an alternative action, here denoted •, of the free monoid $\left(A \cup A^{-1}\right)^{*}$ on terms, namely the action obtained by replacing the basic instance

$$
\left(t_{1} \cdot\left(t_{2} \cdot t_{3}\right)\right) \phi=\left(t_{1} \cdot t_{2}\right) \cdot\left(t_{1} \cdot t_{3}\right)
$$

with

$$
\left(t_{1} \cdot\left(t_{2} \cdot t_{3}\right)\right) \bullet \phi=\left(t_{1} \cdot t_{2}\right) \cdot t_{3} .
$$

Studying the $\bullet$-action leads to introducing new relations, and, therefore, to a new group.

Definition. We denote by $G_{A}$ the group $\left\langle\left\{g_{\alpha} ; \alpha \in A\right\} ; R_{A}\right\rangle$, where $R_{A}$ consists of

$$
\begin{aligned}
g_{\alpha} \cdot g_{\beta} & =g_{\beta} \cdot g_{\alpha} \quad \text { for } \alpha \perp \beta, & (\text { type } \perp) \\
g_{\alpha 0 \beta} \cdot g_{\alpha} & =g_{\alpha} \cdot g_{\alpha 00 \beta}, & (\text { type } 0) \\
g_{\alpha 10 \beta} \cdot g_{\alpha} & =g_{\alpha} \cdot g_{\alpha 01 \beta}, & (\text { type } 10) \\
g_{\alpha 11 \beta} \cdot g_{\alpha} & =g_{\alpha} \cdot g_{\alpha 1 \beta}, & (\text { type } 11) \\
g_{\alpha 1} \cdot g_{\alpha} \cdot g_{\alpha 0} & =g_{\alpha} \cdot g_{\alpha} . & (\text { type } 1)
\end{aligned}
$$

It can now be proved that the (partial) action - of $\left(A \cup A^{-1}\right)^{*}$ on $T_{\infty}$ factors through $G_{A}$, and, if we say that two terms are A-equivalent if we can transform the first into the second using asociativity, we have the following counterpart to Proposition 1.5: 
Proposition 1.6. [6] For every term $t$, the A-equivalence class of $t$ is the orbit of $t$ under the (partial) action of $G_{A}$, and this action is faithful: the term $t^{\prime}$ is $A$-equivalent to $t$ if and only if $t^{\prime}=(t) \bullet$ a holds for some a in $G_{A}$, and, in this case, the involved element a is unique.

Thus, the group $G_{A}$ is an exact counterpart to the group $G_{L D}$. From a technical point of view, the results and the proofs are much easier in the case of associativity because the action is never empty in the latter case.

Proposition 1.7. The group $G_{A}$ is (isomorphic to) Thompson's group F.

Proof. (sketch) One of the standard presentations of $F$ is [1]

$$
\left\langle X_{0}, X_{1}, X_{2}, \ldots ; X_{k}^{-1} X_{n} X_{k}=X_{n+1} \text { for } k<n\right\rangle .
$$

Let us consider the elements $g_{1^{i}}$ in $G_{A}$. An induction on the number of 0's in $\alpha$ shows that, for every address $\alpha, g_{\alpha}$ belongs to the subgroup of $G_{A}$ generated by the $g_{1^{i}}$ 's, i.e., the elements $g_{1^{i}}$ generate $G_{A}$. Moreover, for $k<n$, we have $g_{1^{k}}{ }^{-1} g_{1^{n}} g_{1^{k}}=g_{1^{n+1}}$ by type 11 relations. Hence the mapping $X_{i} \mapsto g_{1^{i}}$ induces a surjective morphism of $F$ onto $G_{A}$. Conversely, for each address $\alpha$, we define an element $Y_{\alpha}$ in $F$ inductively on the number of 0 's in $\alpha$ by $Y_{\alpha}=X_{i}$ for $\alpha=1^{i}$, and

$$
Y_{\alpha}=Y_{\beta}^{-1} Y_{\beta 1}^{-1} \cdots Y_{\beta 1^{k-1}} Y_{\beta 1^{k}}^{-1} Y_{\beta 1^{k+1}}^{-1} Y_{\beta 1^{k}}^{2} Y_{\beta 1^{k-1}} \cdots Y_{\beta 1} Y_{\beta}
$$

for $\alpha=\beta 01^{k}$. The elements $Y_{\alpha}$ satisfy the relations $R_{A}$, so $g_{\alpha} \mapsto Y_{\alpha}$ induces a surjective morphism of $G_{A}$ onto $F$, which is the inverse of the above morphism of $F$ onto $G_{A}$.

Let us mention that a similar approach can be developed for every family of algebraic identities, and refer to [9], where studying the associated group leads to a solution of the word problem of the identity $x(y z)=(x y)(y z)$.

\section{A linear ordering on finite binary trees}

Terms (i.e., finite labeled binary trees) can be equipped with several orderings. Here we consider the linear ordering on $T_{\infty}$ that uses the left height as a discriminant, the latter being defined as the length of the leftmost branch in the associated tree. To make the definition precise, we encode every term by a word and then use a lexicographical ordering.

Definition. For $t$ a term, the left Polish form of $t$ is the word $\llbracket t \rrbracket$ over the alphabet $\left\{x_{1}, x_{2}, \ldots, \bullet\right\}$ defined by the following inductive clauses:

$$
\llbracket t \rrbracket= \begin{cases}t & \text { if } t \text { is a variable } \\ \bullet \llbracket t_{1} \rrbracket \llbracket t_{2} \rrbracket & \text { for } t=t_{1} \cdot t_{2} .\end{cases}
$$


For instance, the left Polish form of the term $x_{1} \cdot\left(x_{2} \cdot x_{3} \cdot x_{4}\right) \cdot x_{5}$ is the word $\bullet x_{1} \bullet \bullet x_{2} \bullet x_{3} x_{4} x_{5}$. When the term $t$ is viewed as a tree, the word $\llbracket t \rrbracket$ is obtained by enumerating the variables of $t$ from left to right and letting each occurrence of a variable be preceded by as many letters • as there are final 0's in the corresponding address. For $w$ a word over the alphabet $\left\{x_{1}, x_{2}, \ldots, \bullet\right\}$, we denote by $\#_{x}(w)$ and \#. $(w)$ the number of letters $x_{i}$ and of letters $\bullet$ in $w$ respectively. By standard arguments, we have the following characterization:

Lemma 2.1. Assume that $w$ is a word over the alphabet $\left\{x_{1}, x_{2}, \ldots, \bullet\right\}$. Then $w$ is the left Polish form of a well formed term if and only if we have $\#_{x}(w)=\# \cdot(w)+1$, and $\#_{x}(u) \leq \#_{.}(u)$ for every proper prefix $u$ of $w$.

Definition. Assume that $t_{1}, t_{2}$ are terms in $T_{\infty}$. We say that $t_{1}<_{L} t_{2}$ holds if the word $\llbracket t_{1} \rrbracket$ precedes the word $\llbracket t_{2} \rrbracket$ in the lexicographical extension of the linear ordering $x_{1}<x_{2}<\cdots<\bullet$.

By construction, the relation $<_{L}$ is a linear ordering on $T_{\infty}$, and $x_{1}$ is minimal for $<_{L}$. If $\mathrm{ht}_{L}(t)$ denotes the left height of the term $t$, the word $\llbracket t \rrbracket$ begins with $\mathrm{ht}_{L}(t)$ letters $\bullet$ followed by a variable. So, $\mathrm{ht}_{L}\left(t_{1}\right)<\mathrm{ht}_{L}\left(t_{2}\right)$ implies $t_{1}<_{L} t_{2}$.

Lemma 2.2. The inequality $t_{1}<_{L} t_{2}$ implies $t_{1} \cdot t_{3}<_{L} t_{2} \cdot t_{4}$ for all terms $t_{3}, t_{4}$.

Proof. Lemma 2.1 implies that a proper prefix of the left Polish form of a term is never the left Polish form of a well formed term. Hence $t_{1}<_{L} t_{2}$ holds if and only if the words $\llbracket t_{1} \rrbracket$ and $\llbracket t_{2} \rrbracket$ have a variable clash of the type "variable $v s$. •". Then the words $\llbracket t_{1} \cdot t_{3} \rrbracket$ and $\llbracket t_{2} \cdot t_{4} \rrbracket$, i.e., $\bullet \llbracket t_{1} \rrbracket \llbracket t_{3} \rrbracket$ and $\bullet \llbracket t_{2} \rrbracket \llbracket t_{4} \rrbracket$, have a similar clash.

We deduce several equivalent characterizations of $<_{L}$.

Lemma 2.3. Assume that $t_{1}, t_{2}$ are terms in $T_{\infty}$. If $t_{1}$ is a variable, say $x_{i}$, then $t_{1}<_{L} t_{2}$ holds unless $t_{2}$ is a variable $x_{j}$ with $j<i$. If $t_{1}$ is not a variable, then $t_{1}<_{L} t_{2}$ holds if and only if either $\operatorname{sub}\left(t_{1}, 0\right)<_{L} \operatorname{sub}\left(t_{2}, 0\right)$ holds, or $\operatorname{sub}\left(t_{1}, 0\right)=$ $\operatorname{sub}\left(t_{2}, 0\right)$ and $\operatorname{sub}\left(t_{1}, 1\right)<_{L} \operatorname{sub}\left(t_{2}, 1\right)$ hold.

Proof. Assume $t_{1}<_{L} t_{2}$, and neither $t_{1}$ nor $t_{2}$ are variables. Three cases are possible. For $\operatorname{sub}\left(t_{1}, 0\right)<_{L} \operatorname{sub}\left(t_{2}, 0\right)$, Lemma 2.2 implies $t_{1}<_{L} t_{2}$. For $\operatorname{sub}\left(t_{1}, 0\right)>_{L}$ $\operatorname{sub}\left(t_{2}, 0\right)$, we obtain $t_{1}>_{L} t_{2}$ symmetrically. Finally, for $\operatorname{sub}\left(t_{1}, 0\right)=\operatorname{sub}\left(t_{2}, 0\right)$, $t_{1}<_{L} t_{2}$ is equivalent to $\operatorname{sub}\left(t_{1}, 1\right)<_{L} \operatorname{sub}\left(t_{2}, 1\right)$ by definition.

In order to state the next result, we need the easy notion of the left edge of an address.

Definition. For $\alpha$ an address, the left edge of $\alpha$ is the finite sequence $\left(\alpha_{1} 0, \ldots, \alpha_{p} 0\right)$, where $\alpha_{1}, \ldots, \alpha_{p}$ are those prefixes of $\alpha$ such that $\alpha_{1} 1, \ldots$, $\alpha_{p} 1$ are prefixes of $\alpha$, enumerated in increasing order. 
For instance, the left edge of 010011 is the sequence $(00,01000,010010)$. As an induction shows, the length of the left edge of the address $\alpha$ is the number of 1's in $\alpha$.

Lemma 2.4. Assume that $t_{1}, t_{2}$ are terms in $T_{\infty}$. Then the following are equivalent:

(i) The relation $t_{1}<_{L} t_{2}$ holds;

(ii) There exists an address $\alpha$ in the skeletons both of $t_{1}$ and of $t_{2}$ such that $\operatorname{sub}\left(t_{1}, \beta\right)=\operatorname{sub}\left(t_{2}, \beta\right)$ holds for every $\beta$ in the left edge of $\alpha$, and $\operatorname{sub}\left(t_{1}, \alpha\right)<_{L}$ $\operatorname{sub}\left(t_{2}, \alpha\right)$ holds.

(iii) There exists an address $\alpha$ both in the outline of $t_{1}$ and in the skeleton of $t_{2}$ such that $\operatorname{sub}\left(t_{1}, \beta\right)=\operatorname{sub}\left(t_{2}, \beta\right)$ holds for every $\beta$ in the left edge of $\alpha$, and either $\operatorname{sub}\left(t_{2}, \alpha\right)$ is a variable larger than $\operatorname{var}\left(t_{1}, \alpha\right)$, or it is not a variable.

Proof. An induction on $\alpha$ shows that, if $\alpha$ belongs to the skeleton of the term $t$ and $\left(\alpha_{1}, \ldots, \alpha_{p}\right)$ is the left edge of $\alpha$, then the word $\llbracket t \rrbracket$ begins with

$$
\bullet^{k_{1}} \llbracket \operatorname{sub}\left(t, \alpha_{1}\right) \rrbracket \bullet^{k_{2}} \llbracket \operatorname{sub}\left(t, \alpha_{2}\right) \rrbracket \ldots \bullet^{k_{p}} \llbracket \operatorname{sub}\left(t, \alpha_{p}\right) \rrbracket \bullet^{k} \llbracket \operatorname{sub}(t, \alpha) \rrbracket,
$$

where $k_{i}$ is the number of final 0's in $\alpha_{i}$ and $k$ is the number of final 0's in $\alpha$. The result is obvious for $\alpha=\phi$, and, otherwise, it follows from an easy induction on $t$. Then, by definition of a lexicographical ordering, it follows from (2.1) and from the fact that a proper prefix of a left Polish form is never the left Polish form of a well formed term that (ii) implies (i).

By construction, (iii) implies (ii). Finally, assuming (i), and letting $\alpha$ be the address of the first position where the words $\llbracket t_{1} \rrbracket$ and $\llbracket t_{2} \rrbracket$ disagree, we obtain (iii) using the explicit expansion of (2.1).

For the next result, we introduce another preordering on terms.

Definition. Assume that $t_{1}, t_{2}$ are terms. We say that $t_{1} \sqsubseteq t_{2}$ holds if and only if $t_{1}$ is an iterated left subterm of $t_{2}$, i.e., $t_{1}=\operatorname{sub}\left(t_{2}, 0^{k}\right)$ holds for some $k \geq 0$. We say that $t_{1} \sqsubseteq_{L D} t_{2}$ holds if there exist two terms $t_{1}^{\prime}, t_{2}^{\prime}$ satisfying $t_{1}^{\prime}=_{L D} t_{1}$, $t_{2}^{\prime}={ }_{L D} t_{2}$, and $t_{1}^{\prime} \sqsubseteq t_{2}^{\prime}$.

It is known [4] that the relation $\complement_{L D}$ induces an ordering on $T_{\infty} /=_{L D}$, whose restriction to $T_{1} /=_{L D}$ is linear.

Lemma 2.5. Assume that $t_{1}, t_{2}$ are $\sqsubseteq_{L D}$-comparable terms in $T_{\infty}$. Then the following are equivalent:

(i) The relation $t_{1}<_{L} t_{2}$ holds;

(ii) There exists an address $\alpha$ in both in the outline of $t_{1}$ and in the skeleton of $t_{2}$ such that $\operatorname{sub}\left(t_{1}, \beta\right)=\operatorname{sub}\left(t_{2}, \beta\right)$ holds for every $\beta$ in the left edge of $\alpha$, and $\operatorname{sub}\left(t_{2}, \alpha\right)$ is a term of left height at least 1 whose leftmost variable is $\operatorname{var}\left(t_{1}, \alpha\right)$. 
Proof. Assume (i). Then there exists an address $\alpha$ satisfying the conditions of Lemma 2.4(iii). We claim that $\operatorname{sub}\left(t_{2}, \alpha\right)$ cannot be a variable. Indeed, assume $\operatorname{sub}\left(t_{1}, \alpha\right)=x_{i}$ and $\operatorname{sub}\left(t_{2}, \alpha\right)=x_{j}$ with $j>i$. Let $\left(\alpha_{1}, \ldots, \alpha_{q}\right)$ denote the left edge of $\alpha$. Let us consider for a while the right Polish form of terms: for $t$ a term, we denote by $[t]$ the word inductively defined by: $[t]=t$ if $t$ is a variable, and $[t]=\left[t_{1}\right]\left[t_{2}\right] \bullet$ for $t=t_{1} \cdot t_{2}$. Then, the word $\left[t_{1}\right]$ begins with $\left[\operatorname{sub}\left(t_{1}, \alpha_{1}\right)\right] \ldots\left[\operatorname{sub}\left(t_{1}, \alpha_{q}\right)\right] x_{i}$, while the word $\left[t_{2}\right]$ begins with $\left[\operatorname{sub}\left(t_{1}, \alpha_{1}\right)\right] \ldots\left[\operatorname{sub}\left(t_{1}, \alpha_{q}\right)\right] x_{j}$. By the results of [4], this is known to contradict the hypothesis that $t_{1}$ and $t_{2}$ are $\sqsubseteq_{L D}$-comparable. So the only possibility is that $\operatorname{sub}\left(t_{2}, \alpha\right)$ is not a variable, and that its leftmost variable is $x_{i}$. This gives (ii). That (ii) implies (i) follows from Lemma 2.4.

The left ordering of terms satisfies several invariance properties. Let us define a substitution to be a mapping of $\left\{x_{1}, x_{2}, \ldots\right\}$ into $T_{\infty}$. If $h$ is a subtitution and $t$ is a term in $T_{\infty}$, we denote by $t^{h}$ the term obtained from $t$ by replacing each variable $x_{i}$ occurring in $t$ with the corresponding term $h\left(x_{i}\right)$. Note that the mapping $t \mapsto t^{h}$ is an endomorphism of the free magma $\left(T_{\infty}, \cdot\right)$, and that every endomorphism of $\left(T_{\infty}, \cdot\right)$ has this form.

Proposition 2.6. Assume that $t_{1}, t_{2}$ are terms in $T_{\infty}$ and $h$ is a substitution of $T_{\infty}$. Assume in addition that at least one of the following conditions holds:

(i) We have $h\left(x_{i}\right)<_{L} h\left(x_{i+1}\right)$ and $\mathrm{ht}_{L}\left(h\left(x_{i}\right)\right)=\mathrm{ht}_{L}\left(h\left(x_{i+1}\right)\right)$ for every $i$;

(ii) The terms $t_{1}$ and $t_{2}$ are $\subseteq_{L D}$-comparable.

Then $t_{1}<_{L} t_{2}$ holds if and only if $t_{1}^{h}<_{L} t_{2}^{h}$ does.

Proof. As $<_{L}$ is a linear ordering, it suffices that we show that $t_{1}<_{L} t_{2}$ implies $t_{1}^{h}<_{L} t_{2}^{h}$. So assume $t_{1}<_{L} t_{2}$. By Lemma 2.4, there exists an address $\alpha$ such that $\operatorname{sub}\left(t_{1}, \beta\right)=\operatorname{sub}\left(t_{2}, \beta\right)$ holds for every $\beta$ in the left edge of $\alpha, \operatorname{sub}\left(t_{1}, \alpha\right)$ is a variable say $x_{i}$, and $\operatorname{sub}\left(t_{2}, \alpha\right)$ is either a variable $x_{j}$ with $j>i$, or it is a term that is not a variable. When Condition (ii) holds, by Lemma 2.5 , we can assume in addition that $\operatorname{sub}\left(t_{2}, \alpha\right)$ is a term with leftmost variable $x_{i}$ and left height at least 1. Applying the substitution $h$, we obtain $\operatorname{sub}\left(t_{1}^{h}, \beta\right)=\operatorname{sub}\left(t_{2}^{h}, \beta\right)$ for every $\beta$ in the left edge of $\alpha$. Then we have $\operatorname{sub}\left(t_{1}^{h}, \alpha\right)=h\left(x_{i}\right)$. Three cases are to be considered.

If Condition (i) holds and we have $\operatorname{sub}\left(t_{2}, \alpha\right)=x_{j}$ with $j>i$, we obtain $\operatorname{sub}\left(t_{2}^{h}, \alpha\right)=h\left(x_{j}\right)>_{L} h\left(x_{i}\right)=\operatorname{sub}\left(t_{1}^{h}, \alpha\right)$. If Condition (i) holds and $\operatorname{sub}\left(t_{2}, \alpha\right)$ is not a variable, the hypothesis on $h$ implies $\mathrm{ht}_{L}\left(\operatorname{sub}\left(t_{2}^{h}, \alpha\right)\right)>\mathrm{ht}_{L}\left(\operatorname{sub}\left(t_{1}^{h}, \alpha\right)\right)$, hence $\operatorname{sub}\left(t_{1}^{h}, \alpha\right)<_{L} \operatorname{sub}\left(t_{2}^{h}, \alpha\right)$. Finally, if Condition (ii) holds and $\operatorname{sub}\left(t_{2}, \alpha\right)$ is a term with leftmost variable $x_{i}$ and left height $k \geq 1$, we find $\mathrm{ht}_{L}\left(\operatorname{sub}\left(t_{1}^{h}, \alpha\right)\right)=$ $\mathrm{ht}_{L}\left(h\left(x_{i}\right)\right)$, and $\mathrm{ht}_{L}\left(\operatorname{sub}\left(t_{2}^{h}, \alpha\right)\right)=\mathrm{ht}_{L}\left(h\left(x_{i}\right)\right)+k$, hence $\operatorname{sub}\left(t_{1}^{h}, \alpha\right)<_{L} \operatorname{sub}\left(t_{2}^{h}, \alpha\right)$. $\operatorname{So}, \operatorname{sub}\left(t_{1}^{h}, \alpha\right)<_{L} \operatorname{sub}\left(t_{2}^{h}, \alpha\right)$ holds in every case. By Lemma 2.4, this implies $t_{1}^{h}<_{L} t_{2}^{h}$.

Definition. For $t$ a term in $T_{\infty}$, we denote by $t^{\dagger}$ the projection of $t$ in $T_{1}$, i.e., 
the image of $t$ under the substitution that maps every variable to $x_{1}$.

Corollary 2.7. (i) Every substitution of $T_{1}$ preserves the ordering $<_{L}$.

(ii) If $t_{1}$ and $t_{2}$ are $\sqsubseteq_{L D}$-comparable terms, $t_{1}<_{L} t_{2}$ is equivalent to $t_{1}^{\dagger}<_{L} t_{2}^{\dagger}$.

Other characterizations of the linear ordering $<_{L}$ can be mentioned. For instance, if we assume that $t, t_{1}, t_{2}$ are terms and the outline of $t$ is included in the skeleton of $t_{1}$ and $t_{2}$, then, letting $\left(\alpha_{1}, \ldots, \alpha_{p}\right)$ be the left-right enumeration of the outline of $t, t_{1}<_{L} t_{2}$ holds if and only if the sequence $\left(\operatorname{sub}\left(t_{1}, \alpha_{1}\right), \ldots, \operatorname{sub}\left(t_{1}, \alpha_{p}\right)\right)$ precedes the sequence $\left(\operatorname{sub}\left(t_{2}, \alpha_{1}\right), \ldots, \operatorname{sub}\left(t_{2}, \alpha_{p}\right)\right)$ in the lexicographical extension of $<_{L}$ to $T_{\infty}^{*}$.

In the special case of $T_{1}$, it can also be checked that $t_{1}<_{L} t_{2}$ holds if and only if the left-right increasing enumeration of the outline of $t_{1}$ precedes the left-right increasing enumeration of the outline of $t_{2}$ with respect to the lexicographical extension of the prefix ordering of addresses to $A^{*}$.

\section{The linear ordering on $G_{L D}$}

We use now the partial action of the group $G_{L D}$ on the linearly ordered set $\left(T_{\infty},<_{L}\right)$ to define a linear ordering on $G_{L D}$. The ordering so defined has nice properties, in particular it is compatible with multiplication on both sides, so $G_{L D}$ is a biorderable group.

The first step is to prove that the action of $G_{L D}$ on $T_{\infty}$ preserves the ordering $<_{L}$.

Proposition 3.1. For all terms $t_{1}, t_{2}$ in $T_{\infty}$, and every a in $G_{L D}$ such that $\left(t_{1}\right) a$ and $\left(t_{2}\right) a$ exist, $t_{1}<_{L} t_{2}$ holds if and only if $\left(t_{1}\right) a<_{L}\left(t_{2}\right) a$ does.

Proof. As $G_{L D}$ is generated by the elements $g_{\alpha}$ with $\alpha \in A$, it suffices to prove the result for the latter elements, i.e., to prove that, if $\alpha$ is an address, and $t_{1}$, $t_{2}$ are terms then $t_{1}<_{L} t_{2}$ is equivalent to $\left(t_{1}\right) \alpha<_{L}\left(t_{2}\right) \alpha$ when the latter terms are defined. As the action of $\alpha$ is injective, it suffices to prove that $t_{1}<_{L} t_{2}$ implies $\left(t_{1}\right) \alpha<_{L}\left(t_{2}\right) \alpha$. We use induction on $\alpha$. Assume first that $\alpha$ is the empty address. The hypothesis that $\left(t_{1}\right) \phi$ and $\left(t_{2}\right) \phi$ exist implies that $\operatorname{sub}\left(t_{e}, 0\right), \operatorname{sub}\left(t_{e}, 10\right)$, and $\operatorname{sub}\left(t_{e}, 11\right)$ exist for $e=1,2$, and we have the explicit decompositions

$$
\llbracket t_{e} \rrbracket=\bullet \llbracket \operatorname{sub}\left(t_{e}, 0\right) \rrbracket \bullet \llbracket \operatorname{sub}\left(t_{e}, 10\right) \rrbracket \llbracket \operatorname{sub}\left(t_{e}, 11\right) \rrbracket,
$$

and

$$
\llbracket\left(t_{e}\right) \lambda \rrbracket=\bullet \bullet \llbracket \operatorname{sub}\left(t_{e}, 0\right) \rrbracket \llbracket \operatorname{sub}\left(t_{e}, 10\right) \rrbracket \bullet \llbracket \operatorname{sub}\left(t_{e}, 0\right) \rrbracket \llbracket \operatorname{sub}\left(t_{e}, 11\right) \rrbracket .
$$

By Lemma 2.3, only three cases are possible, namely

$-\operatorname{sub}\left(t_{1}, 0\right)<_{L} \operatorname{sub}\left(t_{2}, 0\right)$, or

$-\operatorname{sub}\left(t_{1}, 0\right)=\operatorname{sub}\left(t_{2}, 0\right)$ and $\operatorname{sub}\left(t_{1}, 10\right)<_{L} \operatorname{sub}\left(t_{2}, 10\right)$, or 
$-\operatorname{sub}\left(t_{1}, 0\right)=\operatorname{sub}\left(t_{2}, 0\right), \operatorname{sub}\left(t_{1}, 10\right)=\operatorname{sub}\left(t_{2}, 10\right)$ and $\operatorname{sub}\left(t_{1}, 11\right) \quad<_{L}$ $\operatorname{sub}\left(t_{2}, 11\right)$,

and the result is clear in each case.

Assume now $\alpha=0 \beta$. Then we have $\left(t_{e}\right) \alpha=\operatorname{sub}\left(t_{e}, 0\right) \beta \cdot \operatorname{sub}\left(t_{e}, 1\right)$. Two cases are possible. For $\operatorname{sub}\left(t_{1}, 0\right)<_{L} \operatorname{sub}\left(t_{2}, 0\right)$, by induction hypothesis, we have $\operatorname{sub}\left(t_{1}, 0\right) \beta<_{L} \operatorname{sub}\left(t_{2}, 0\right) \beta$, and, therefore, $\left(t_{1}\right) \alpha<_{L}\left(t_{2}\right) \alpha$. For $\operatorname{sub}\left(t_{1}, 0\right)=$ $\operatorname{sub}\left(t_{2}, 0\right)$ and $\operatorname{sub}\left(t_{1}, 1\right)<_{L} \operatorname{sub}\left(t_{2}, 1\right)$, we have $\operatorname{sub}\left(t_{1}, 0\right) \beta=\operatorname{sub}\left(t_{2}, 0\right) \beta$, and, again, $\left(t_{1}\right) \alpha<_{L}\left(t_{2}\right) \alpha$.

Assume finally $\alpha=1 \beta$. Then we have $\left(t_{e}\right) \alpha=\operatorname{sub}\left(t_{e}, 0\right) \cdot \operatorname{sub}\left(t_{e}, 1\right) \beta$. Two cases are possible again. For $\operatorname{sub}\left(t_{1}, 0\right)<_{L} \operatorname{sub}\left(t_{2}, 0\right)$, we deduce $\left(t_{1}\right) \alpha<_{L}\left(t_{2}\right) \alpha$ directly. For $\operatorname{sub}\left(t_{1}, 0\right)=\operatorname{sub}\left(t_{2}, 0\right)$ and $\operatorname{sub}\left(t_{1}, 1\right)<_{L} \operatorname{sub}\left(t_{2}, 1\right)$, the latter inequality implies $\operatorname{sub}\left(t_{1}, 1\right) \beta<_{L} \operatorname{sub}\left(t_{2}, 1\right) \beta$ by induction hypothesis, and we deduce $\left(t_{1}\right) \alpha<_{L}\left(t_{2}\right) \alpha$ again.

Lemma 3.2. Assume that a belongs to $G_{L D}^{+} \backslash\{1\}$. Then $t<_{L}(t)$ a holds whenever $(t) a$ is defined.

Proof. It suffices to consider the case of a single address $\alpha$. If $\alpha$ is the empty address, the result follows from the equality $\mathrm{ht}_{L}((t) \phi)=\mathrm{ht}_{L}(t)+1$. Otherwise, we use an induction on $\alpha$, or simply resort to Lemma 2.4: by the previous argument, we have $\operatorname{sub}(t, \alpha)<_{L} \operatorname{sub}((t) \alpha, \alpha)$, and, by construction, $\operatorname{sub}(t, \beta)=\operatorname{sub}((t) \alpha, \beta)$ holds for every $\beta$ in the left edge of $\alpha$.

The next step consists in using the action of the submonoid $G_{L D}^{+}$of $G_{L D}$ on $T_{\infty}$ to order $G_{L D}^{+}$. For each element $a$ of $G_{L D}^{+}$, we shall need a characterization of those terms $t$ for which $(t) a$ is defined. Let us say that a term $t$ is canonical if the list of all variables that occur in $t$, enumerated from left to right ignoring repetitions, is an initial segment of $\left(x_{1}, x_{2}, \ldots\right)$. The following result is proved in [3] (in a general framework).

Proposition 3.3. Assume that $a_{1}, \ldots, a_{k}$ are elements of $G_{L D}^{+}$. Then there exists a unique canonical term $t_{L}\left(a_{1}, \ldots, a_{k}\right)$ such that, for every term $t$, the terms $(t) a_{1}, \ldots,(t) a_{k}$ all are defined if and only if $t=t_{L}\left(a_{1}, \ldots, a_{k}\right)^{h}$ holds for some substitution $h$.

Lemma 3.4. For $a, b \in G_{L D}^{+}$, the following are equivalent:

(i) There exists a term $t$ in $T_{\infty}$ such that $(t) a<_{L}(t) b$ holds;

(ii) The inequality $\left(t_{L}(a, b)\right) a<_{L}\left(t_{L}(a, b)\right) b$ holds;

(iii) For every term $t$ in $T_{\infty}$ such that $(t) a$ and $(t) b$ exist, $(t) a<_{L}(t) b$ holds.

Proof. That (ii) implies (i) and (iii) implies (ii) is clear. So assume (i). By construction, there exists a substitution $h$ satisfying $t=t_{L}(a, b)^{h}$, and our hypothesis is the inequality $\left(t_{L}(a, b)^{h}\right) a<_{L}\left(t_{L}(a, b)^{h}\right) b$, i.e., $\left(\left(t_{L}(a, b)\right) a\right)^{h}<_{L}\left(\left(t_{L}(a, b)\right) b\right)^{h}$. The terms $\left(t_{L}(a, b)\right) a$ and $\left(t_{L}(a, b)\right) b$ are LD-equivalent, hence, by Proposition 2.6, 
the previous inequality is equivalent to $\left(t_{L}(a, b)\right) a<_{L}\left(t_{L}(a, b)\right) b$, which gives (ii), and, then, to $\left(\left(t_{L}(a, b)\right) a\right)^{g} \quad<_{L}\left(\left(t_{L}(a, b)\right) b\right)^{g}$ for every substitution $g$, which gives (iii).

Definition. For $a, b \in G_{L D}^{+}$, we say that $a<b$ holds if the equivalent conditions of Lemma 3.4 are satisfied.

Proposition 3.5. The relation $<$ is a linear ordering on the monoid $G_{L D}^{+}$that is compatible with multiplication on both sides; it admits 1 as a minimal element.

Proof. That the relation $<$ is irreflexive is clear as $<_{L}$ is an ordering on terms. Assume $a<b<c$, and let $t$ be a term such that $(t) a,(t) b$, and $(t) c$ are defined, for instance $t=t_{L}(a, b, c)$. By Lemma 3.4, $a<b$ implies $(t) a<_{L}(t) b$, and $b<c$ implies $(t) b<_{L}(t) c$. We deduce $(t) a<_{L}(t) c$, which in turn gives $a<c$ by Lemma 3.4. So $<$ is an ordering on $G_{L D}^{+}$, and it is linear as $<_{L}$ is a linear ordering on $T_{\infty}$.

Assume now $a<b$, and let $c$ be an arbitrary element of $G_{L D}^{+}$. Let $t$ be a term such that both $(t) c a$ and $(t) c b$ exist. By construction, we have $(t) c a=((t) c) a$ and $(t) c b=((t) c) b$, so the hypothesis $a<b$ implies $((t) c) a<_{L}((t) c) b$, which in turn implies $c a<c b$ by definition. With the same hypotheses, assume that $(t) a c$ and $(t) b c$ are defined. Then $(t) a<(t) b$ holds by hypothesis, and, by Proposition 3.1, this implies $((t) a) c<_{L}((t) b) c$, which in turn implies $a c<b c$ by definition. Finally, assume $a \neq 1$. By Lemma 3.2, $t<_{L}(t) a$ holds, so, by definition, we have $1<a$.

It is now easy to extend the ordering of $G_{L D}^{+}$to the whole of $G_{L D}$.

Lemma 3.6. For $a, b, a^{\prime}, b^{\prime} \in G_{L D}^{+}$satisfying $a b^{-1}=a^{\prime} b^{-1}, a<b$ is equivalent to $a^{\prime}<b^{\prime}$.

Proof. By Proposition 1.1, there exist $c, c^{\prime}$ in $G_{L D}^{+}$satisfying $a c=a^{\prime} c^{\prime}$ and $b c=b^{\prime} c^{\prime}$. Assume $a<b$. Using the compatibility of the order with multiplication on the right, we deduce $a c<b c$, i.e., $a^{\prime} c^{\prime}<b^{\prime} c^{\prime}$, hence $a^{\prime}<b^{\prime}$.

Definition. For $c, d \in G_{L D}$, we say that $c<d$ holds if $c d^{-1}=a b^{-1}$ holds for some $a, b$ in $G_{L D}^{+}$satisfying $a<b$.

Proposition 3.7. The relation $<$ is a linear order on the group $G_{L D}$ that extends the order $<$ on $G_{L D}^{+}$. This order is compatible with multiplication on both sides, and, therefore, it is compatible with conjugacy.

Proof. For $a, b$ in $G_{L D}^{+}, 1=a b^{-1}$ implies $a=b$, hence $a \nless b$, hence, for every $c$ in $G_{L D}, c<c$ is impossible. Assume $c<d<e$ in $G_{L D}$. There exist $a_{1}, b_{1}, a_{2}, b_{2}$ in $G_{L D}^{+}$satisfying $c d^{-1}=a_{1} b_{1}^{-1}, d e^{-1}=a_{2} b_{2}^{-1}, a_{1}<b_{1}$, and $a_{2}<b_{2}$. Let $a_{3}, b_{3}$ be elements of $G_{L D}^{+}$satisfying $a_{2} b_{3}=b_{1} a_{3}$. We find

$$
c e^{-1}=a_{1} b_{1}^{-1} a_{2} b_{2}^{-1}=\left(a_{1} a_{3}\right)\left(b_{2} b_{3}\right)^{-1} .
$$


The hypothesis $a_{1}<b_{1}$ implies $a_{1} a_{3}<b_{1} a_{3}$, the hypothesis $a_{2}<b_{2}$ implies $a_{2} b_{3}<b_{2} b_{3}$. By hypothesis, we have $b_{1} a_{3}=a_{2} b_{3}$, so we deduce $a_{1} a_{3}<b_{2} b_{3}$, and, therefore, $c<e$. Hence the relation $<$ is an ordering on $G_{L D}$.

Assume $a, b \in G_{L D}^{+}$and $a<b$ holds in the sense of $G_{L D}^{+}$. Then $a b^{-1}$ is an expression of $a b^{-1}$ with $a, b$ in $G_{L D}^{+}$and $a<b$, i.e., $a<b$ in the sense of $G_{L D}$ holds. Thus the order $<$ on $G_{L D}$ extends the previous order $<$ on $G_{L D}^{+}$.

Assume now $c, d, e \in G_{L D}$ and $c<d$. By definition, there exist $a, b$ in $G_{L D}^{+}$ satisfying $c d^{-1}=a b^{-1}$ and $a<b$. Then we have $(c e)(d e)^{-1}=a b^{-1}$, so $c e<d e$ holds as well. On the other hand, let us express $e$ as $a_{0} b_{0}^{-1}$ with $a_{0}, b_{0}$ in $G_{L D}^{+}$. There exist $a_{1}, b_{1}, a_{2}, b_{2}, a_{3}, b_{3}$ in $G_{L D}^{+}$satisfying $b_{0} a_{1}=a a_{2}, b_{0} b_{1}=b b_{2}, a_{2} a_{3}=b_{2} b_{3}$ (Figure 3.1). Then, we find

$$
(e c)(e d)^{-1}=a_{0} b_{0}^{-1} a b^{-1} b_{0} a_{0}^{-1}=\left(a_{0} a_{1} a_{3}\right)\left(a_{0} b_{1} b_{3}\right)^{-1} .
$$

The hypothesis $a<b$ implies $b_{0} a_{1} a_{3}=a a_{2} a_{3}<b a_{2} a_{3}=b b_{2} b_{3}=b_{0} b_{1} b_{3}$, whereas we deduce $a_{1} a_{3}<b_{1} b_{3}$, and, therefore, $a_{0} a_{1} a_{3}<a_{0} b_{1} b_{3}$ using compatibility with multiplication on the left twice. By (3.1), this gives $e c<e d$.

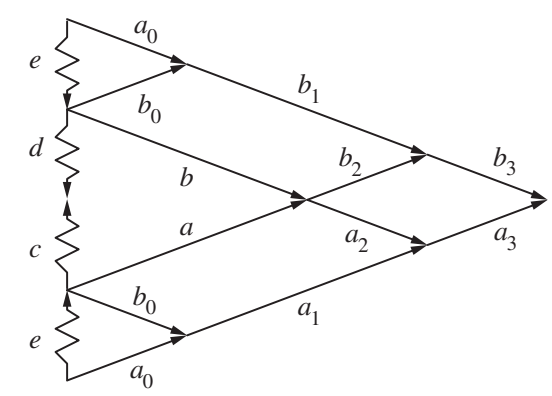

Figure 3.1. Compatibility of order with multiplication on the left.

We thus have proved our main result, namely that $G_{L D}$ is a bi-orderable group. By general results [18], we deduce

Corollary 3.8. The group $G_{L D}$ is torsion free, the group algebra $\mathbf{C} G_{L D}$ admits no zero divisor, and it embeds in a skew field.

The action of the group $G_{L D}$ on terms is a partial action. In particular, some elements of $G_{L D}$ do not act, i.e., the domain of the associated operator is empty. Hence, we cannot compare all elements of $G_{L D}$ using their action on terms directly. However, using the action gives a sufficient condition when it is defined.

Proposition 3.9. (i) Assume $c, d \in G_{L D}$ and there exists a term $t$ such that $(t) c$ and $(t) d$ are defined. Then $c<d$ holds in $G_{L D}$ if and only if $(t) c<_{L}(t) d$ holds in $T_{\infty}$. 
(ii) Assume $c \in G_{L D}$ and there exists at least one term $t$ such that $(t) c$ exists. Then $c>1$ holds in $G_{L D}$ if and only if $(t) c>_{L} t$ holds for any term $t$ such that $(t) c$ exists.

Proof. (i) Assume that $t$ is a term and $(t) c$ and $(t) d$ are defined. By Proposition 1.1, there exist $a$ and $b$ in $G_{L D}^{+}$satisfying $c^{-1} d=a b^{-1}$, hence $c a=d b$. We cannot claim that $(t) c a$ is defined in general, but, as a belongs to $G_{L D}^{+}$, the only possible obstruction for $(t) c a$ to be defined is the skeleton of $(t) c$ being too small. Now, for every substitution $h$, the term $t^{h}$ is eligible for the action of $c$ as well as $t$ is, and we can choose $h$ such that the skeleton of $\left(t^{h}\right) c$ is arbitrary large. Then $\left(t^{h}\right) c a$ exists, so does $\left(t^{h}\right) d b$, and we have $\left(t^{h}\right) c a=\left(t^{h}\right) d b$. Assume now $c<d$, hence $a>b$. We deduce $(t) c b<_{L}(t) c a=(t) d b$, and, therefore, $(t) c<_{L}(t) d$. The argument is symmetric for $c>d$.

Point (ii) follows by taking $d=1$ in (i).

\section{Action of $G_{L D}^{+}$on the Cantor space and the reals}

We conclude the paper with the observation that the previous action of the monoid $G_{L D}^{+}$on finite binary trees induces an action on the Cantor space, viewed as a line at infinity for the set $A$ of all binary addresses.

We first introduce a partial action of $G_{L D}^{+}$on addresses by using the origin function. The idea is that, if $a$ is an element of $G_{L D}^{+}$and $t$ is a term large enough to make sure that $(t) a$ exists, then every address $\beta$ in the skeleton of $(t) a$ has a well-defined origin in the skeleton of $t$. A direct definition can be posed easily.

Definition. Assume that $\alpha, \beta$ are addresses. The origin $\alpha(\beta)$ of $\beta$ under $\alpha$ is defined by

$$
\alpha(\beta)= \begin{cases}\beta & \text { if } \beta \perp \alpha \text { holds or } \alpha 11 \text { is a prefix of } \beta, \\ \alpha 0 \gamma & \text { for } \beta=\alpha 00 \gamma \text { and } \beta=\alpha 10 \gamma, \\ \alpha 10 \gamma & \text { for } \beta=\alpha 01 \gamma \\ \text { undefined } & \text { if } \beta \text { is a prefix of } \alpha 1 .\end{cases}
$$

Lemma 4.1. (i) Defining $\alpha_{1} \cdot \ldots \cdot \alpha_{k}(\beta)$ to be $\alpha_{1}\left(\ldots\left(\alpha_{p}(\beta) \ldots\right)\right.$ induces a partial left action of $G_{L D}^{+}$on $A$.

(ii) For $a \in G_{L D}^{+}$, denote by a $(\beta)$ the image of $\beta$ under the action of $a$, when it exists. Then $a(\beta)$ is defined if and only if some prefix $\beta^{\prime}$ of $\beta$ lies in the outline of the term $t_{L}(a)$, and, in this case, we have $a(\beta)=a\left(\beta^{\prime}\right) \gamma$ where $\beta$ is $\beta^{\prime} \gamma$.

(iii) If $t$ is a term with pairwise distinct variables, then, for every address $\beta$ in the skeleton of $(t) a$, the address a $(\beta)$ is the unique address in the skeleton of $t$ such that the variable occurring at $\beta$ in $(t) a$ is the variable occurring at $a(\beta)$ in $t$. 
The easy verifications are left to the reader.

Remark. We have switched from a right action to a left action here because the origin function actually goes backwards: we could have considered instead the inheriting function that associates with every address in the skeleton of a term $t$ its heirs in the term $(t) a$. Inheriting corresponds to a right action, but, in contradistinction to the case of associativity, it does not define a function on addresses, as a given address may have several heirs: for instance, the heir of the address 0 under the action of $\phi$ consists of the two addresses 00 and 10, since the variable $x$ in $x(y z)$ has two copies at 00 and 10 respectively in $(x y)(x z)$. However, inheriting is injective, and we obtain a function by considering its inverse, which is the current origin function.

The action of $G_{L D}^{+}$on $A$ is partial: by Lemma 4.1(ii), for each $a$ in $G_{L D}^{+}, a(\beta)$ is defined only when $\beta$ is long enough, i.e., it does not lie in some neighbourhood of $\phi$ for the topology $\mathcal{T}$ on $A$ associated with the distance defined by $d(\alpha, \beta)=2^{-n}$ if $\alpha \neq \beta$ holds and $n$ is the length of the greatest common prefix of $\alpha$ and $\beta$. Now, by Lemma 4.1(ii) again, the action is $\mathcal{T}$-continuous on $A$, so we can extend it into an everywhere defined action on the $\mathcal{T}$-boundary of $A$, which is the Cantor line $\widehat{A}$ consisting of all $\mathbf{N}$-indexed sequences of 0's and 1's.

Definition. For $s \in \widehat{A}$ and $a \in G_{L D}^{+}$, the element $a(s)$ of $\widehat{A}$ is defined to be $a(\beta) s_{0}$, where $\beta$ is the unique prefix of $s$ lying in the outline of $t_{L}(a)$ and $s=\beta s_{0}$ holds.

By Lemma 4.1, the previous action is defined everywhere. The reader can easily check the equalities $g_{\phi}(000 \cdots)=g_{\phi}(100 \cdots)=000 \cdots, g_{\phi}(00111 \cdots)=$ $g_{\phi}(10111 \cdots)=0111 \cdots, g_{\phi}(01000 \cdots)=1000 \cdots$. More generally, the action of $g_{\phi}$ on $\widehat{A}$ is displayed on Figure 4.1 .

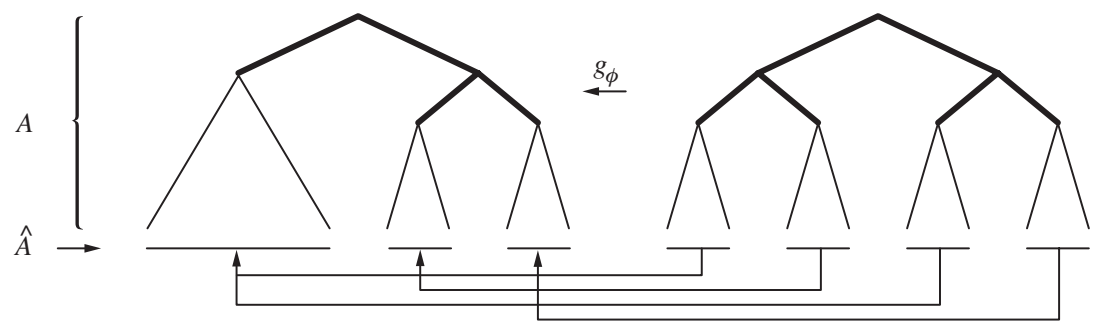

Figure 4.1. Action of $G_{L D}^{+}$on the Cantor set.

Let us equip $\widehat{A}$ with the lexicographical ordering, which corresponds to the usual ordering of dyadic numbers, and with the associated topology.

Proposition 4.2. For every a in $G_{L D}^{+}$, the action of a on $\widehat{A}$ is surjective, it is continuous on the right, and it admits finitely many left discontinuities. 
Proof. The result is clear for every generator $\gamma_{\alpha}$, and it is preserved under multiplication.

The linear order of $G_{L D}^{+}$can be defined in terms of the action of $G_{L D}^{+}$on the Cantor line $\widehat{A}$ :

Proposition 4.3. The action of $G_{L D}^{+}$on $\widehat{A}$ preserves the order in the sense that $a<b$ holds in $G_{L D}^{+}$if and only if there exists $s_{0}$ in $\widehat{A}$ such that $a(s)=b(s)$ holds for $s \leq s_{0}$, but $a(s)<b(s)$ holds for $s>s_{0}$, s close enough to $s_{0}$.

Proof. Assume $a<b$ in $G_{L D}^{+}$. Let $t$ be a term with pairwise distinct variables such that $(t) a$ and $(t) b$ exist. By Lemma 3.4, we have $(t) a<(t) b$, hence, as $(t) a$ and $(t) b$ are LD-equivalent, Lemma 2.5 tells us that there exists an address $\alpha$ such that $\alpha$ lies in the outline of $t_{1}$ and in the skeleton of $t_{2}, \operatorname{sub}((t) a, \beta)=\operatorname{sub}((t) b, \beta)$ holds for every $\beta$ in the left edge of $\alpha$ (hence for every $\beta$ on the left of $\alpha$ such that the considered subterms exist $), \operatorname{sub}((t) a, \alpha)$ is a variable say $x_{i}$, and $\operatorname{sub}((t) b, \alpha)$ is a term of size at least 2 whose leftmost variable is $x_{i}$. Let $s_{0}$ be $\alpha 000 \cdots$. By construction, $a(s)=b(s)$ holds for $s \leq s_{0}$. In particular, we have $a\left(s_{0}\right)=b\left(s_{0}\right)=$ $\gamma 000 \cdots$, where $\gamma$ is the address where $x_{i}$ occurs in $t$. Let $q$ be the left height of the term $\operatorname{sub}((t) b, \alpha)$. For $p \geq q$, we have

$$
a\left(\alpha 0^{p} 1000 \cdots\right)=\gamma 0^{p} 1000 \cdots, \quad \text { and } \quad b\left(\alpha 0^{p} 1000 \cdots\right)=\gamma 0^{p-q} 1000 \cdots
$$

Hence we have $a(s)<b(s)$ for points $s$ arbitrarily close on the right of $s_{0}$. As the action is continuous on the right, this is enough to conclude.

Finally, we can copy the previous left action of $G_{L D}^{+}$on $\widehat{A}$ into an action on the real interval $[0,1)$ using the dyadic expansion. This amounts to associating with every element $a$ of $G_{L D}^{+}$a piecewise affine mapping $f_{a}$ of $[0,1)$ into itself. For instance, $f_{\phi}$ is defined by

$$
f_{\phi}(x)= \begin{cases}2 x & \text { for } 0 \leq x<1 / 4 \\ x+1 / 4 & \text { for } 1 / 4 \leq x<1 / 2 \\ 2 x-1 & \text { for } 1 / 2 \leq x<3 / 4 \\ x & \text { for } 3 / 4 \leq x<1\end{cases}
$$

In Figure 4.2 we have displayed the function $f_{\phi}$ associated with the action of left self-distributivity at $\phi$, i.e., at the root of the tree, and its counterpart when associativity replace self-distributivity (when compared with the diagrams of [12], the current diagram is inversed because we consider the origin function). Similarly, we have represented in Figure 4.3 the rectangle diagrams associated with a few positive words both in the case of associativity, as in [1], and left self-distributivity. 

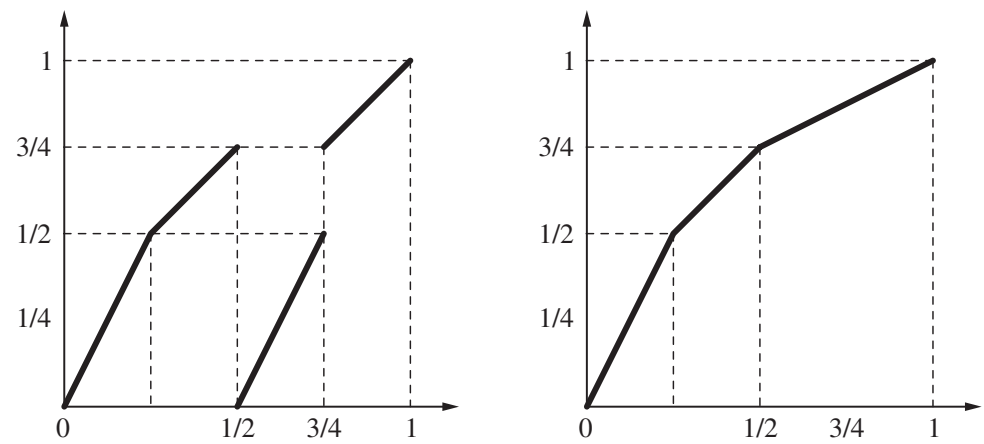

Figure 4.2. Left self-distributivity vs. associativity: action at $\phi$ on the reals.

action of $\phi$ :

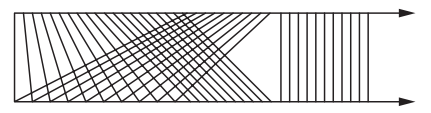

action of 1:

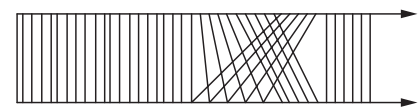

action of 0 :

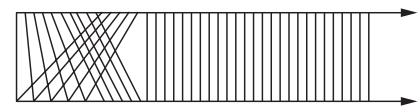

action of $\phi \cdot \phi$ :

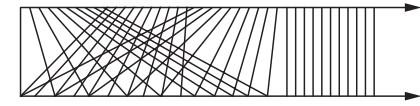

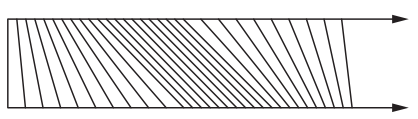
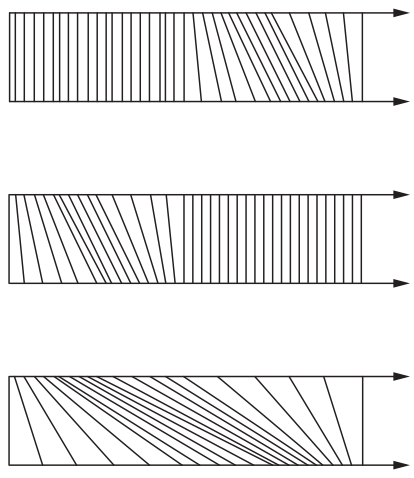

Figure 4.3. Left self-distributivity vs. associativity: rectangle diagrams.

Using Proposition 4.3, we deduce:

Proposition 4.4. The relation $a<b$ holds in $G_{L D}^{+}$if and only if there exists a real $x_{0}$ satisfying $a(x)=b(x)$ for $x \leq x_{0}$ and $a\left(x_{0}+\varepsilon\right)<b\left(x_{0}+\varepsilon\right)$ for $\varepsilon$ small enough.

In the case of associativity, using the previous approach amounts to defining the action of Thompson's group $F$ on the reals considered in [12]. Then the mappings $f_{a}$ are bijections, the action is defined on the group, and not only on the monoid, the counterpart of Proposition 4.4 is straightforward, and we obtain a linear ordering on $F$ trivially. In the case of self-distributivity, the result is not so easy, for some form of the nontrivial result expressed in Lemma 2.5 is required. 


\section{References}

[1] J. W. Cannon, W. J. Floyd, W. R. Parry, Introductory notes on Richard Thompsons's groups, Ens. Math. 42 (1996), 215-257.

[2] P. Dehornoy, Free distributive groupoids, J. P. Appl. Algebra 61 (1989), 123-146.

[3] P. Dehornoy, Structural monoids associated to equational varieties, Proc. Amer. Math. Soc. 117-2 (1993), 293-304.

[4] P. Dehornoy, Braid groups and left distributive operations, Trans. Amer. Math. Soc. 345-1 (1994), 115-151.

[5] P. Dehornoy, Groups with a complemented presentation, J. Pure Appl. Algebra 116 (1997), 115-137.

[6] P. Dehornoy, The structure group for the associativity identity, J. Pure Appl. Algebra 111 (1996), 59-82.

[7] P. Dehornoy, A fast method for comparing braids, Advances in Math. 125 (1997), 200-235.

[8] P. Dehornoy, The geometry monoid of left self-distributivity, preprint.

[9] P. Dehornoy, Study of an identity, preprint.

[10] R. Fenn, M. T. Greene, D. Rolfsen, C. Rourke, B. Wiest, Ordering the braid groups, Pacific J. of Math., to appear.

[11] F. A. Garside, The braid group and other groups, Quart. J. Math. Oxford 20 No.78 (1969), 235-254.

[12] E. Ghys, V. Sergiescu, Sur un groupe remarquable de difféomorphismes du cercle, Comment. Math. Helvetici 62 (1987), 185-239.

[13] C. Kassel, L'ordre de Dehornoy sur les tresses, Séminaire Bourbaki, exposé 865 (novembre 1999).

[14] D. M. Kim, D. Rolfsen, An ordering of pure braids and hyperplane arrangements, Preprint.

[15] R. Laver, Braid group actions on left distributive structures and well-orderings in the braid group, J. Pure Appl. Algebra 108-1 (1996), 81-98.

[16] S. Mac Lane, Natural associativity and commutativity, Rice Univ. Studies 49 (1963), $28-46$

[17] R. McKenzie, R. J. Thompson, An elementary construction of unsolvable word problems in group theory, in Word Problems, Boone \& al. eds., North Holland, Studies in Logic vol. 71 (1973), 457-478.

[18] D. S. Passman, The Algebraic Structure of Group Rings, Pure and Appl. Math., Wiley Interscience, 1977.

[19] A. Rhemtulla, D. Rolfsen, Incompatible group orderings, local indicability and braids, preprint.

[20] C. Rourke, B. Wiest, Order automatic mapping class groups, Pacific J. Math., to appear.

[21] H. Short, B. Wiest, Ordering the mapping class groups after Thurston, preprint.

[22] R. J. Thompson, Embeddings into finitely generated simple groups which preserve the word problem, in Word problems II: The Oxford book, S.I. Adjan \& al. eds., Studies in Logic vol. 95, North Holland (1980), 401-440. 
Patrick Dehornoy

Laboratoire SDAD

Département de mathématiques

Université de Caen, Campus II

F-14032 Caen

France

e-mail: dehornoy@math.unicaen.fr

(Received: September 10, 1999, revised version: June 5, 2000) 